\title{
COVID-19 Pandemic: Effects on the Dental Team and Implications on Dental Public Health
}

\author{
Anne-Marie Agius $^{1} \odot$, Gabriella Gatt ${ }^{\circledR}$, ${\text { Arthur RG } \text { Cortes }^{3} \odot \text {, Nikolai J Attard }}^{4}$ (이
}

\begin{abstract}
Aim and objective:To assess the self-reported impact of coronavirus disease-2019 (COVID-19) pandemic on the different members of the dental team in public, private, and academic sectors in Malta.

Materials and methods: An anonymous online questionnaire on self-reported work and lifestyle changes was designed and administered. Since data were classified as categorical ranks, nonparametric tests were used to compare variables among the different dental team members. Statistical analysis: Multiple-choice question and checklist responses were treated as qualitative variables and were compared among groups using the Chi-square test. Questionnaire data obtained from the Likert scale, in turn, were treated as categorical ranks and therefore assessed using the Kruskal-Wallis test. Results from all variables obtained from the questionnaire were compared among the different dental team members. A $p<0.05$ significance level was used for all the tests.

Results: Dental hygienists were significantly more anxious most of the time during the pandemic $(p=0.004)$. Furthermore, dental hygienists and associate dentists were significantly more anxious about contracting COVID-19 themselves $(p=0.005, p=0.001)$ or one of their family members $(p=0.024$ and $p=0.001)$. Both were also more anxious about not being able to work as much as they used to $(p=0.070, p=0.008)$. Dental clinic owners presented significantly fewer work changes than associates $(p<0.001)$.

Conclusion: All dental team members were affected by this pandemic regarding anxiety due to health concerns, financial burdens as well as abrupt work, and lifestyle changes. However, dental hygienists were significantly more affected by the pandemic than any of the other dental team members.

Keywords: Coronavirus, Dentistry, Lifestyle, Stress factors, Work changes.

Journal of Oral Health and Community Dentistry (2021): 10.5005/jp-journals-10062-0106
\end{abstract}

\section{INTRODUCTION}

Coronavirus disease-2019 (COVID-19) pandemic has brought about many changes in people's lifestyle, work, and relationships. Due to the high rate of transmission of severe acute respiratory syndrome coronavirus 2 (SARS-CoV-2), severe social distancing measures were introduced, including countrywide lockdowns, closing borders, asking people to stop visiting family members, and closing down of entertainment outlets including restaurants, bars, music/drama events, and the like.

Most workplaces were also impacted to some extent, and this is especially true for healthcare facilities. Despite the great recent advances in medical/dental equipment and stringent crossinfection control measures in place in hospitals and clinics prior to the pandemic, this was still not enough to protect staff and patients from COVID-19, and hospitals and clinics had to undertake major overhauls to be able to safely treat patients again. ${ }^{1,2}$

The dental team was tremendously impacted as the nature of dental treatment puts the team at the highest risk of contracting and transmitting COVID-19., ${ }^{3,4}$ Transmission rates and routes of SARS-CoV-2 and possible transmission by asymptomatic COVID-19-positive patients made it impossible to safely treat patients routinely especially when the dental treatment provided generated aerosols - this occurring in most dental procedures. ${ }^{5,6}$ Emerging evidence suggests that without adequate air ventilation or filtration systems, aerosolized viral particles can remain suspended in the air for long periods of time. Such particles can be inhaled by anyone present in the room during and after treatment until eventually, they settle down on all objects in the room. ${ }^{3}$
${ }^{1,4}$ Department of Oral Rehabilitation and Community Care, Faculty of
Dental Surgery, University of Malta, Msida, Malta
${ }^{2}$ Department of Child Dental Health and Orthodontics, Faculty of
Dental Surgery, University of Malta, Msida, Malta
${ }^{3}$ Department of Dental Surgery, Faculty of Dental Surgery, University ${ }^{3}$ Department of Dent
of Malta, Msida, Malta

Corresponding Author: Anne-Marie Agius, Department of Oral Rehabilitation and Community Care, Faculty of Dental Surgery, University of Malta, Msida, Malta, Phone: +35623401216 , e-mail: anne-marie.agius@um.edu.mt

How to cite this article: Agius AM, Gatt G, Cortes ARG, et al. COVID-19 Pandemic: Effects on the Dental Team and Implications on Dental Public Health. J Oral Health Comm Dent 2021;15(2):64-69.

Source of support: Nil

Conflict of interest: None

These revelations brought about drastic changes both in the short and long term. Guidance documents were issued from several bodies advising against aerosol-generating procedures (AGPs) where possible while trying to provide timely urgent dental treatment safely with the use of personal protective equipment (PPE). ${ }^{7-9}$ Also present in the literature are a number of articles and systematic reviews about transmission routes and measures to be taken at dental clinics and about what needs to change for safely performing AGPs. ${ }^{5,10,11}$

In Malta, the dental community is small and composed mainly of dentists. In the last published document, there were 230

() The Author(s). 2021 Open Access This article is distributed under the terms of the Creative Commons Attribution 4.0 International License (https://creativecommons. org/licenses/by-nc/4.0/), which permits unrestricted use, distribution, and non-commercial reproduction in any medium, provided you give appropriate credit to the original author(s) and the source, provide a link to the Creative Commons license, and indicate if changes were made. The Creative Commons Public Domain Dedication waiver (http://creativecommons.org/publicdomain/zero/1.0/) applies to the data made available in this article, unless otherwise stated. 
registered dentists in Malta (36\% female), 100 dental assistants, and 21 registered dental hygienists. ${ }^{12}$ These numbers have now increased slightly since the latest published document in 2015. Most dentists work in private practice with a fee-per-item remuneration that patients pay out of pocket. The services provided for free for the population are preventive and emergency services. Restorative treatments are only provided for people who are on social benefits or diabetic patients. As a result, most people have to seek treatment privately and dental insurances are still uncommon in Malta. ${ }^{13}$

Little is known and documented, however, about the impact that the pandemic had on the different dental team members who on one hand are stressed about the possibility of contracting and transmitting SARS-CoV-2 and on the other hand, understand that timely dental emergency treatment is vital and that they also have financial obligations to fulfil. In this context, standardized questionnaires of self-reported stress currently available are not able to assess the wider effects of the pandemic on dental team members, which include lifestyle changes, dealing with financial obligations, retaining staff members, and coping with the pandemic.

Thus, the aim of this study was to assess the self-reported impact of the COVID-19 pandemic on the different members of the dental team, working in private clinics, in public service, and at university.

\section{Materials and Methods}

\section{Sample}

This cross-sectional population study was conducted in Malta, on different members of the dental team working in public, private health sectors, and at the University of the Country. All participants consented to the use of data derived from the completed questionnaires. This study was approved by the local Ethics Committee (Protocol number: DSG/2019-2020/009). The STROBE guidelines for cross-sectional studies and the Helsinki Declaration guidelines were also carefully observed during the research. Failing to submit the questionnaire was the only exclusion criterion. The data were collected between May 19, and May 30, 2020.

\section{Questionnaire}

To address the wider effects of the pandemic, an original anonymous questionnaire consisting of nine questions was developed to assess the various impacts of the pandemic on the different dental team members (Table 1). Some of the questions were similar to the DASS-21 questionnaire ${ }^{14}$ and were modified to address the impact of the pandemic on the dental teams' lifestyle, work, and financial obligations. Five close-ended multiple-choice questions, two checklist-type questions, and two Likert-scale-style questions were included. ${ }^{15}$ The personal data collected could not identify specific individuals. This included gender and work description. After gathering required permissions, the questionnaire was first piloted and some minor changes were made, and then it was sent to the local dental associations and the public health unit as a link to an anonymous online questionnaire hosted by Google Forms. These were in turn distributed to all the members.

Responses were automatically gathered into Google Forms, and a data sheet was generated in Microsoft Excel. These data were then

Table 1: COVID-19 questionnaire for dental team members

\begin{tabular}{|c|c|c|}
\hline Category & Questions & Type of answers \\
\hline \multirow[t]{2}{*}{ Demographics } & Gender & Multiple-choice answer \\
\hline & Choose which descriptions fit your work as a dental professional & Multiple-choice answer \\
\hline \multirow[t]{3}{*}{ Current work } & What is your current clinical work situation? & Multiple-choice answer \\
\hline & $\begin{array}{l}\text { Thinking now about patients coming to your clinical workplace at the present. } \\
\text { Which of the following measures have you implemented or plan to implement? } \\
\text { (tick all that apply) }\end{array}$ & Checklist with answers \\
\hline & $\begin{array}{l}\text { If a patient in severe pain is potentially infected with COVID-19 (answered yes to } \\
\text { symptom questions/recent contact with COVID-19 case/has fever) how do you } \\
\text { plan to deal with the situation? }\end{array}$ & Multiple-choice answer \\
\hline Financial obligations & $\begin{array}{l}\text { If you are a practice owner, do you currently have issues with meeting your business } \\
\text { financial obligations such as employee wages, clinic rent, and clinic loans? }\end{array}$ & Likert scale type: $1-5$ \\
\hline \multirow[t]{2}{*}{ Lifestyle changes } & Have you had to make any major lifestyle changes because of your work? & Yes/no \\
\hline & If yes, what changes did you make? & Checklist answer \\
\hline \multirow{9}{*}{$\begin{array}{l}\text { Mental and emotional } \\
\text { well-being }\end{array}$} & I feel anxious most of the time & Likert scale \\
\hline & I am anxious about contracting COVID-19 & Likert scale \\
\hline & I am worried that one of my family members contracts COVID-19 & Likert scale \\
\hline & This has affected my personal relationship with my partner & Likert scale \\
\hline & This has affected my relationships with family & Likert scale \\
\hline & This has affected my relationships with friends and work colleagues & Likert scale \\
\hline & I worry about not being able to work as much as I used to & Likert scale \\
\hline & I feel obliged to work somewhere else to alleviate my financial burdens & Likert scale \\
\hline & I don't like not knowing how and when this will end & Likert scale \\
\hline
\end{tabular}


cleaned and coded. A reminder was sent to the participants after 5 days but no incentives were offered to participate in the study.

\section{Statistical Analyses}

The minimum sample size was previously calculated with the uncorrected Chi-square to give the study a statistical power of $80 \%$ within a significance level of $5 \%$. Multiple-choice question and checklist responses were treated as qualitative variables and were compared among groups using the Chi-square test. Questionnaire data obtained from the Likert scale, in turn, were treated as categorical ranks and therefore assessed using the Kruskal-Wallis test.

Results from all variables obtained from the questionnaire were compared among the different dental team members [i.e., dental technologists (DT), dental hygienists (DH), dental surgery assistants (DSA), and dental surgeons (DS)]. Additional comparisons were also assessed between dentists who are dental clinic owners vs associate dentists, academic vs nonacademic participants and gender.

A $p<0.05$ significance level was used for all the tests. All statistical analyses were performed with the same software (SPSS 22.0; SPSS Inc., Chicago, Illinois, United States).

\section{Results}

\section{Dental Team Members}

The questionnaire was sent out to 324 participants from different associations, namely the Dental Association of Malta and Dental Public Health Unit (243 members), the Dental Surgery Assistants Association of Malta (44 dental surgery assistants), and the Malta Association of Dental Hygienists (31 dental hygienists). This questionnaire was sent out to a quasi-total population of dental team members in this small country. A total of 105 members of the dental team completed the questionnaire. The highest number of participants were dentists $69.6 \%(n=73)$. This was expected as there are many more dentists registered in this country than any other member of the dental team. There are 13 dental hygienist participants and 17 dental surgery assistant participants in the study.

The vast majority of participants has been impacted by the pandemic and has had to make significant changes to their work environment, type of patients seen, and to their personal lifestyle. Only four participants answered that they were still seeing all patients with dental disease. About $68 \%(n=70)$ of participants were seeing only urgent or true emergency cases, while $25.2 \%$ $(n=26)$ stopped seeing and treating patients completely (Fig. 1).

Statistically significant differences were found between those dental team members still treating patients and those having stopped treating patients. All dental hygienist respondents $(n=12)$ stated that they are not treating patients at all, while all dental clinic owners reported still treating patients $(n=41)$.

The remaining question responses were similar for the different team members. Most participants $(70.5 \%, n=74)$ reported making major lifestyle changes because of their work during the pandemic. The most common change reported was "not meeting family and friends," selected by $72.7 \%$ of the participants who answered "yes" to major lifestyle changes. Moreover, $11.7 \%$ of participants moved out of their homes because of vulnerable family members within the household, while a further $20.8 \%$ isolated themselves from their family members within the same household.

Participants were also asked which measures they implemented already or were willing to implement to be able to treat patients. The most common measures chosen by more than $70 \%$ of respondents were "take patient temperature on arrival," "allow only patient to attend (no accompanying persons, except with children)," and "keep patients waiting off premises until you are ready to see them." "Call patients a day in advance and ask if they have symptoms of COVID-19" was chosen by $65.6 \%$ of participants while only $23.7 \%$ would request patients to take a preoperative COVID-19 nasal swab. Just over half of the respondents stated that if a patient who is potentially COVID-19 positive is in severe pain, they would prescribe analgesics and antibiotics and treat the patient when the patient recovers (Fig. 2).

The final set of questions in the questionnaire was about the participants' emotional well-being and stressors during the pandemic. Several significant differences were found in the responses of participants in different groups. Hygienists were significantly more anxious ( $p=0.04$ ) and hygienists and associate dentists were also more anxious than clinic owners with most choosing agree or strongly agree to the statement "I am anxious most of the time" ( $p=0.09$ and $p=0.014$, respectively). Hygienists and associate dentists were also significantly more worried about contracting COVID-19 themselves ( $p=0.005$ and $p=0.001$, respectively, (Fig. 3). Both groups were also worried about one of their family members contracting COVID-19 ( $p=0.024$ and $p=0.001$, respectively). The same groups were also more anxious about not being able to work as much as they used to previously ( $p=0.070$ and $p=0.008$, respectively) considering to work elsewhere to alleviate their financial burdens $(p=0.013)$. Dental assistants were the least concerned with not being able to work as much as they used to ( $p<0.001$, (Fig. 4). Responses differed significantly between all dental team members with regard to their relationship with their partner being affected due to the pandemic. Dental clinic owners reported being the least affected by this $(p=0.012)$.

\section{Dental Clinic Owner vs Associate}

When practice owners were asked whether they have any difficulty meeting their business financial obligations, there was a good number, $36.9 \%(n=21)$, who reported that they have "bad-to-severe financial issues" choosing 4 or 5 from a Likert scale of $1-5$ with 5 being "severe financial issues."

Practice owners and associates differed significantly with regard to their stand on treating patients $(p=0.000)$ with none of the dental clinic owners stopping to practice completely.

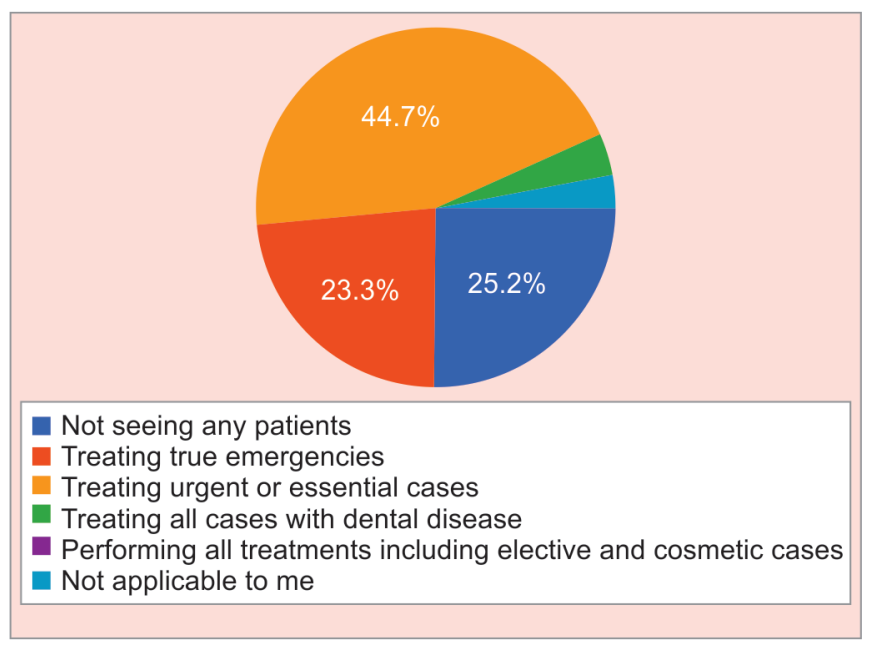

Fig. 1: Current clinical situation of all participants 


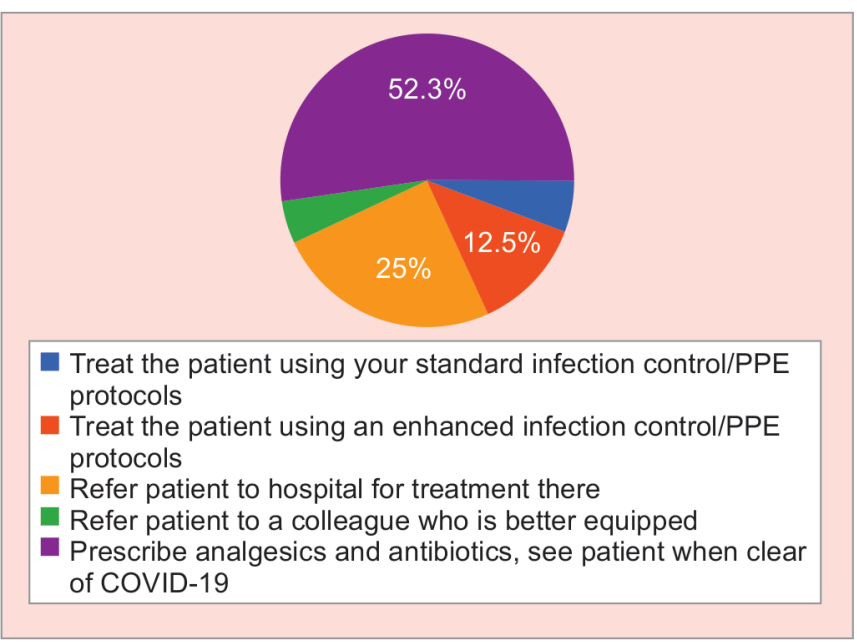

Fig. 2: Management of a potentially COVID-19-positive patient

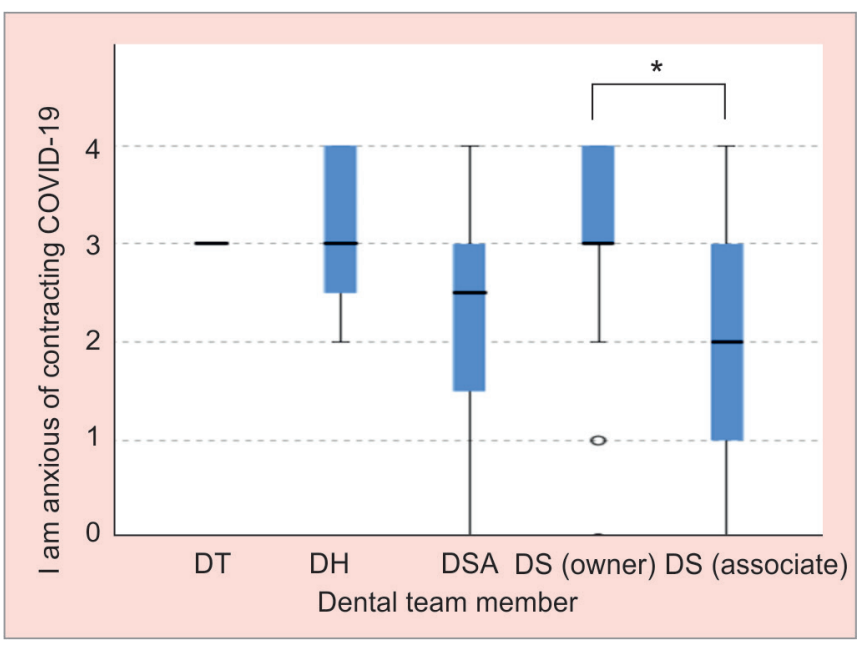

Fig. 3: Fear of contracting COVID-19 among different dental team members (Kruskal-Wallis $p=0.003$ ). ${ }^{*}$ Significant pairwise difference $(p<0.05)$

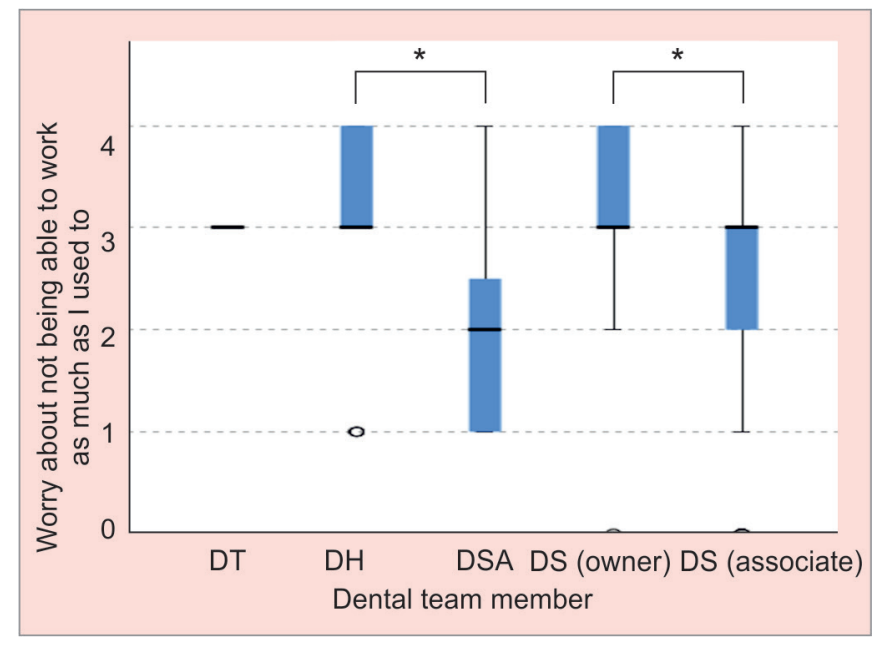

Fig. 4: Worry about not being able to work as much as before in different dental team members (Kruskal-Wallis $p=0.002$ ). ${ }^{*}$ Significant pairwise difference $(p<0.05)$
Associates were also stressed about working less but are less willing to risk infection than clinic owners.

\section{Academic vs Nonacademic}

There were only four full-time academics and seven part-time academic respondents. When academic vs nonacademic groups were compared, responses were very similar with the only significant difference being their current working situation such that $45 \%$ of academics were not seeing patients at the clinic $(p=0.001)$.

\section{Gender}

Gender comparisons resulted in significant differences in the current working situation where more females stopped seeing patients during the pandemic when compared to males ( $p=0.022)$. Female participants were also significantly more anxious about contracting COVID-19 $(p=0.006)$ and also felt more obliged to try and work somewhere else for financial reasons $(p=0.009)$.

When asked about the number of measures currently being undertaken or planned measures to prevent contracting COVID-19 at their clinic when treating patients, females reported taking more measures than males ( $p=0.014)$.

\section{Discussion}

This cross-sectional study aimed at assessing the self-reported outcomes of the impact of the COVID-19 pandemic as well as any professional and lifestyle changes. Results indicated that many of the participants were considerably affected by the pandemic and had to make significant changes to their work, workplace, and their personal life. The time period of this questionnaire reflects a time period when some COVID-19 measures were being lifted in the country of this study. There still were, however, protection requirements and social distancing restrictions in place. Due to the small population of the country, the present results can be considered as representative of group of all dental professionals and are in accordance with a previous study stating changes in oral health services due to COVID-19. ${ }^{1-3}$

Besides the abovementioned general findings, this is the first study addressing differences in the impact of the pandemic among dental team members. In this context, the most severely affected herein were dental hygienists, who entirely stopped seeing patients during the pandemic. Studies show that as dental hygiene practice routinely involves the use of ultrasonic instruments, thereby AGPs lead to a higher risk of COVID-19 transmission. Furthermore, the nature of their work precludes them from using rubber dam isolation and treatment directly involves soft tissues, generating blood, and saliva-aerosolized particles. Hand instrumentation, therefore, is one of a few work alternatives for dental hygienists during the pandemic. Dental hygienists and associate dentists reported the highest anxiety levels with regard to not being able to work as much as they used to. Both of the aforementioned groups work for clinic owners and therefore are not the primary decisionmakers. In addition, these same groups reported significantly higher anxiety levels regarding fear of themselves or a family member contracting COVID-19.

More than $70 \%$ of the participants reported that, in agreement with the local published guidelines, they were taking the necessary measures before treating patients. This is in accordance with a previous study in the literature. ${ }^{4}$ Most of the participants chose four out of five measures offered in the questionnaire. Among the 
answers, the least chosen by participants was "taking a preoperative nasal swab," which is in agreement with the lack of acceptance of swabbing by dental patients described in the literature. ${ }^{16}$ For this reason, the aforementioned citation also suggests saliva-based PCR tests as an alternative to swabbing for dental patients. On the contrary, there were a quarter of the participants who still planned to implement this measure in the future.

Participants were also very hesitant about treating a potentially COVID-19-positive patient in severe pain, with the vast majority stating that they would either postpone treatment until the patient is healthy or refer him/her to the hospital for treatment. This could be due to the participants not feeling well-equipped with adequate $\mathrm{PPE}^{4,10,11}$ and/or adequate clinic ventilation systems to treat potentially-positive/positive patients safely.

Dental surgery assistants were the group that was least concerned about not working as much as before. However, it is pertinent to note that most of the participants in this study are assistants who work in public service or university as their primary job. This means that their job security and salary were not greatly affected by the pandemic. For a similar reason, academics have reported seeing significantly fewer patients during the peak of the pandemic, which could be probably as a result of needing to focus on the sudden changes happening at the university, ensuring their salary as faculty members. As expected, however, most practice owners had to keep treating patients to make sure that they cater for patient needs but also to fulfil their financial obligations that had been severely impacted. Clinic owners also reported being the least affected by relationships with their partners. This could be due to them already being established and living with their partners for a while before the pandemic, and there was a little perceived change in the relationship.

This is also the first study to report significant differences between genders regarding self-reported stress due to the pandemic. In the present results, female dental team members reported more anxiety and fear of contracting COVID-19 themselves and one of their family members and also reported taking more measures to combat COVID-19 at their workplaces than male counterparts. More females stopped working than males and consequently reported more anxiety about not earning as much as before. This may be explained by the fact that schools in the country of this study were also closed at the time of the pandemic and several females who are mothers felt compelled to stay home with their children.

This pandemic brought about changes in work protocols and clinic designs as well as personal lives of all dental team members. The pandemic is leading to changes in the present and future of dentistry, and it will be challenging to practice safely unless major overhauls are made. On the contrary, several routine and technical changes are currently being made in dental clinics to address these concerns as the pandemic develops further; future longterm studies would be recommended to determine the benefits of those changes in the practice of dental team members in their self-reported stress outcomes.

Despite performing a sample size calculation, the major limitation of this study is the small sample size, even though the questionnaire was sent out to all DT, DH, DSA, and DS in the country. The numbers, however, reflect the size of this country, which is small, geographically isolated, and has a unique context.

\section{Conclusion}

All dental team members were affected by this pandemic, and this brought about a lot of anxiety due to health concerns, financial burdens, and abrupt changes. Postponing routine treatments, checkups, and preventive measures until after the pandemic will have significant implications on the oral health of communities worldwide, increasing the burden of disease with a toll on health, economy, and quality of life.

\section{Clinical Relevance}

\section{Scientific Rationale}

COVID-19 pandemic is affecting healthcare professionals. However, little is known about differences in the impact that the pandemic had among different dental team members.

\section{Principal Findings}

Dental team members are anxious and stressed about the pandemic. Dental hygienists are significantly more anxious and feel most at risk doing AGPs.

\section{Practical Implications}

Better PPE should be provided and clinic facilities should be improved so that dental team members can safely practice routine dentistry, especially dental hygienists who cannot use rubber dam for AGPs. Psychological support should be considered to support the dental team members to deal with their anxiety.

\section{ACKnOWLedgments}

The authors thank the Malta Association of Dental Hygienists, the Dental Association of Malta, Dental Public Health Unit, and the Dental Surgery Assistants Association of Malta, for distributing the questionnaires to their members.

\section{ORCID}

Anne-Marie Agius (1) https://orcid.org/0000-0002-7138-860X

Gabriella Gatt @ https://orcid.org/0000-0003-1721-4374

Arthur RG Cortes @ https://orcid.org/0000-0001-6591-7256

Nikolai J Attard @ https://orcid.org/0000-0002-0742-9665

\section{References}

1. Ather A, Patel B, Ruparel NB, et al. Coronavirus disease 19 (COVID-19): implications for clinical dental care. J Endod 2020;46(5):584-595. DOI: 10.1016/j.joen.2020.03.008.

2. Meng L, Hua F, Bian Z. Coronavirus disease 2019 (COVID-19): emerging and future challenges for dental and oral medicine. J Dent Res 2020;99(5):481-487. DOI: 10.1177/0022034520914246.

3. Peng X, Xu X, Li Y, et al. Transmission routes of 2019-nCoV and controls in dental practice. Int J Oral Sci 2020;12(1):9. DOI: 10.1038/s41368-0200075-9.

4. Izzetti R, Nisi M, Gabriele M, et al. COVID-19 transmission in dental practice: brief review of preventive measures in Italy. J Dent Res 2020;99(9):1030-1038. DOI: 10.1177/0022034520920580.

5. Ren YF, Rasubala L, Malmstrom H, et al. Dental care and oral health under the clouds of COVID-19. JDR Clin Trans Res 2020;5(3):202-210. DOI: $10.1177 / 2380084420924385$.

6. Wu KY, Wu DT, Nguyen TT, et al. COVID-19's impact on private practice and academic dentistry in North America. Oral Dis 2021;27(Suppl. 3):684-687. DOI: 10.1111/odi.13444.

7. Cochrane Oral Health Database. COVID-19 (coronavirus): resources for the oral and dental care team. 2020. Available from: https:// oralhealth.cochrane.org/news/covid-19-coronavirus-resources-oraland-dental-care-team [Accessed April 25, 2020].

8. General Dental Council. COVID-19 latest information. 2020. Available from: https://www.gdc-uk.org/information-standards-guidance/ covid-19/covid-19-latest-information [Accessed September 04, 2020]. 
9. Centres for Disease Control and Prevention. CDC guidance for providing dental care during COVID-19.2020. Available from: https:// www.cdc.gov/coronavirus/2019-ncov/hcp/dental-settings.html [Accessed September 04, 2020].

10. Jouhar R, Naseer A, Adnan S, et al. Fear and practice modifications among dentists to combat novel coronavirus disease (COVID-19) outbreak. Int J Environ Res Public Health 2020;17(8):2821. DOI: 10.3390/ijerph17082821.

11. Cortes ARG, Galea K, No-Cortes J, et al. Use of free CAD design software for 3D printing individualized face masks based on face scans [einsatz von CAD-design-freeware zur individualisierung 3-D-gedruckter atemschutzmasken anhand von gesichtsscans]. Int J Comput Dent 2020;23(2):1-7. PMID: 32337516.

12. Kravitz AS, Bullock A, Cowpe J, et al. Council of European dentists, manual of dental practice 2015, 5.1th ed. 2015. Available from:
https://www.eoo.gr/files/pdfs/enimerosi/EU_Manual_of_Dental_ Practice_2015_Edition_5.1.pdf [Accessed September 04, 2020].

13. Azzopardi-Muscat N, Buttigieg S, Calleja N, et al. Malta: health system review. Health Syst Transit 2017;19(1):1-137. Available from: http:// www.euro.who.int/en/about-us/partners/obser

14. Talaee N, Varahram M, Jamaati $\mathrm{H}$, et al. Stress and burnout in health care workers during COVID-19 pandemic: validation of a questionnaire. J Public Health 2020:1-6. DOI: 10.1007/s10389-02001313-z. Available from: https://doi.org/10.1007/s10389-020-01313-z

15. Attard N, Schembri A, Caruana C, et al. Undergraduate students' evaluation and reflections on a gerodontology programme. Eur J Dent Educ 2018;22(3):e624-e633. DOI: 10.1111/eje.12367.

16. Takeuchi Y, Furuchi M, Kamimoto A, et al. Saliva-based PCR tests for SARS-CoV-2 detection. J Oral Sci 2020;62(3):350-351. DOI: 10.2334/ josnusd.20-0267. 